\title{
Is there a relationship between different types of prior bariatric surgery and post-thyroidectomy hypocalcemia?
}

\author{
Benedetto Cali $^{1} \wedge$, Ariola Hasani ${ }^{1} \wedge$, Camille Buffet $^{2,3} \wedge$, Fabrice Menegaux ${ }^{1,3}$, Nathalie Chereau ${ }^{1,3} \wedge$ \\ ${ }^{1}$ Department of General and Endocrine Surgery, Pitié Salpêtrière Hospital, APHP, Sorbonne University, 47-83 Boulevard de l'Hôpital, Paris, France; \\ ${ }^{2}$ Thyroid and Endocrine Tumor Unit, Pitié Salpêtrière Hospital, APHP, Sorbonne University, 47-83 Boulevard de l'Hôpital, Paris, France; ${ }^{3}$ Groupe \\ de Recherche Clinique n 16 Thyroid Tumors, Sorbonne University, Paris, France \\ Contributions: (I) Conception and design: B Cali, N Chereau, A Hasani; (II) Administrative support: C Buffet, F Menegaux, A Hasani; (III) Provision \\ of study materials or patients: F Menegaux, N Chereau, C Buffet; (IV) Collection and assembly of data: B Cali, A Hasani; (V) Data analysis and \\ interpretation: N Chereau, A Hasani; (VI) Manuscript writing: All authors; (VII) Final approval of manuscript: All authors. \\ Correspondence to: Ariola Hasani, MD. 47-83 Boulevard de l'Hôpital, 75013, Paris, France. Email: ariolahasani@gmail.com.
}

\begin{abstract}
Background: Hypocalcemia is a common complication after total thyroidectomy (TT). A history of bariatric surgery has been identified as a risk factor for this complication. This study aimed to assess the risk of hypocalcemia post TT in patients with a history of obesity procedures: laparoscopic sleeve gastrectomy (LSG), Roux-en-Y gastric bypass (RYGB), and laparoscopic gastric banding (LAGB).

Methods: We compared the risk of hypocalcemia post TT (serum calcium levels $<8 \mathrm{mg} / \mathrm{dL}$ ) between patients with restrictive (LSG and LAGB), malabsorptive (RYGB), and patients without a history of obesity surgery. Hypoparathyroidism was considered permanent if the plasma parathyroid hormone (PTH) levels at 6 months were less than $15 \mathrm{pg} / \mathrm{mL}$ (normal range: $15-65 \mathrm{pg} / \mathrm{mL}$ ) and the patient still required oral calcium (calcium carbonate) and vitamin D supplementation, in addition to the supplements that were taken routinely before thyroidectomy.

Results: From the 13,242 patients who underwent TT from 2006 to 2018,90 patients (0.7\%) had a history of bariatric surgery: 35 LAGB, 29 LSG, and 26 RYGB. The risk of hypocalcemia was higher in RYGB patients $(50 \%, \mathrm{n}=13)$ than in LAGB $(17.1 \%, \mathrm{n}=6)$ or LSG patients $(20.6 \%, \mathrm{n}=6)(\mathrm{P}=0.003)$. Furthermore, hypocalcemia risk was similar between patients with a history of restrictive procedures $(18.8 \%, 12 / 64)$ and patients with no history of bariatric surgery $(17.2 \%, 2,268 / 13,152)(\mathrm{P}=0.4)$. Permanent hypoparathyroidism was observed in one and 6 patients from the LAGB and RYGB groups, respectively; however, it was not observed in any patient from the LSG group.
\end{abstract}

Conclusions: RYGB is a risk factor for hypocalcemia post TT, while restrictive bariatric procedures are not.

Keywords: Bariatric surgery; hypocalcemia; thyroidectomy; gastric bypass; restrictive procedure

Submitted Apr 06, 2021. Accepted for publication Jun 23, 2021.

doi: 10.21037 /gs-21-225

View this article at: https://dx.doi.org/10.21037/gs-21-225

\footnotetext{
^ ORCID: Benedetto Cali, 0000-0003-0934-8153; Ariola Hasani, 0000-0002-4086-9320; Camille Buffet, 0000-0003-1389-4157; Fabrice Menegaux, 0000-0003-4337-251X; Nathalie Chereau, 0000-0002-9706-3133.
} 


\section{Introduction}

Obesity, a global public health problem, is a major risk factor for several conditions including cardiovascular diseases, diabetes, musculoskeletal disorders, and some cancers $(1,2)$.

While bariatric surgery is considered the most effective therapy for morbid obesity (3), it may lead to malnutrition, irrespective of a malabsorptive or restrictive procedure. It is associated with several vitamin and mineral deficiencies, which often do not respond to oral supplements (3-6). For instance, $15-48 \%$ of bariatric surgery patients experience low serum calcium levels, while $30-60 \%$ have vitamin D deficiency $(7,8)$.

Total thyroidectomy (TT) is commonly performed for either malignant or benign thyroid diseases (9). Hypocalcemia is a relatively frequent complication of TT $(15-20 \%)$, with a permanent hypoparathyroidism rate of $1-3 \%(10,11)$. Certain clinical and biochemical risk factors for postoperative hypocalcemia have been identified. These include female sex, autoimmune and inflammatory thyroid diseases (Hashimoto thyroiditis or Graves' disease), and bilateral central neck dissection for malignancies $(11,12)$. However, the role of bariatric surgery as a risk factor remains debatable (13-20).

Considering the increasing prevalence of obesity in the western world and the rising number of different bariatric procedures, documenting risk factors for hypocalcemia after TT in this cohort is important to prevent and/or treat its complications in them.

Therefore, this study aimed to assess the risk of hypocalcemia following thyroidectomy in patients with a history of various bariatric procedures such as laparoscopic sleeve gastrectomy (LSG), Roux-en-Y gastric bypass (RYGB), or laparoscopic gastric banding (LAGB). We present the following article in accordance with the STROBE reporting checklist (available at https://dx.doi. org/10.21037/gs-21-225).

\section{Methods}

All patients with a history of bariatric surgery who underwent TT at our University Hospital from 2006 to 2018 were included in this study. Our department is a highvolume center specialized in endocrine surgery, with 1,500 thyroidectomies per year.

Patients with a history of thyroid, parathyroid, or other cervical surgeries were excluded.
The study was conducted in accordance with the Declaration of Helsinki (as revised in 2013).

The study was approved by institutional review board of Assistance Publique-Hôpitaux de Paris (APHP) (IRB number 20200115171338) and informed consent was taken from all the patients.

Data on the demographic, biochemical, and preoperative characteristics [age, sex, serum calcium and vitamin D levels, and body mass index (BMI) at the time of thyroidectomy], along with data on postoperative hypocalcemia, were retrospectively analyzed from the prospectively completed database.

Postoperative hypocalcemia was defined as a serum calcium level of less than $8 \mathrm{mg} / \mathrm{dL}$ (normal range: $8.4-$ $10.6 \mathrm{mg} / \mathrm{dL}$ ) during the postoperative course after TT that was either symptomatic with overt manifestations (such as anxiety, carpopedal spasms, tingling, or numbness of the extremities) or not.

In the event of hypocalcemia, $1.25 \mathrm{~g}$ of oral calcium carbonate was administered thrice daily as a calcium supplement; in case of severe symptoms, calcium was administered intravenously. Calcium was supplemented with vitamin D (alfacalcidol: $1 \mu \mathrm{g} /$ day) if the serum calcium level was less than $7.2 \mathrm{mg} / \mathrm{dL}$. The nadir serum calcium level, defined as the lowest concentration of calcium at any time after thyroidectomy, was recorded.

Hypoparathyroidism was considered permanent if the plasma parathyroid hormone (PTH) levels at 6 months were less than $15 \mathrm{pg} / \mathrm{mL}$ (normal range: $15-65 \mathrm{pg} / \mathrm{mL}$ ) and the patient still required oral calcium (calcium carbonate) and/or vitamin D supplementation, in addition to the supplements that were taken routinely before thyroidectomy. In our clinical practice, we follow a perioperative supplementation protocol for patients undergoing TT; briefly, patients receive $0.25 \mu \mathrm{g} /$ day of alfacalcidol for 5 days prior to the thyroidectomy.

This protocol was applied to all patients, including bariatric patients, who underwent TT. Postoperatively, at discharge, calcium supplementation was based on the PTH levels (dosed 20 minutes after thyroidectomy).

Our TT technique involved identifying the parathyroid glands (especially the superior glands) for their preservation. If the four parathyroid glands were not observed during the dissection, the resected specimen was examined for the missing glands at the end of the procedure. Patients with compromised parathyroid gland vascularization underwent selective parathyroid auto-transplantation into the ipsilateral sternocleidomastoid muscle. 


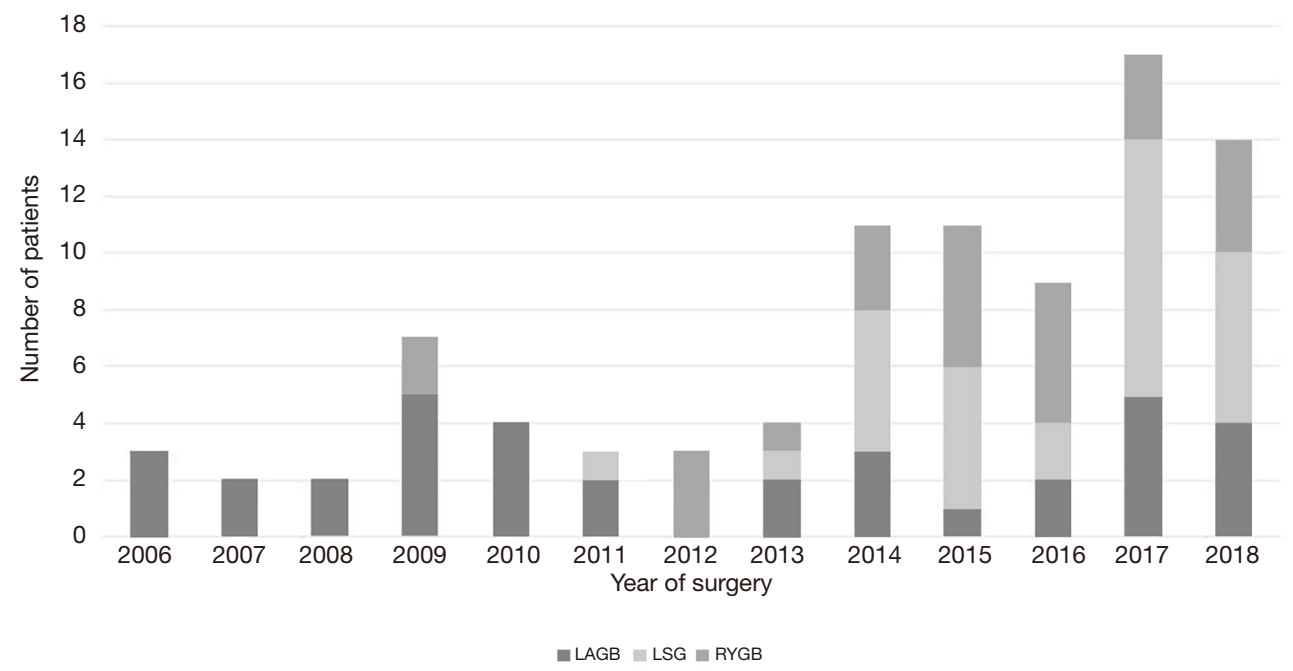

Figure 1 Incidence of patients with a history of bariatric surgery undergoing TT. LAGB, laparoscopic gastric banding; LSG, laparoscopic sleeve gastrectomy; RYGB, Roux-en-Y gastric bypass; TT, total thyroidectomy.

The follow-up consisted of an outpatient visit 6 weeks after surgery; patients who developed hypocalcemia were seen by their surgeon after 6 and 12 months.

To identify the transient and definitive effects of previous bariatric surgery on postoperative hypocalcemia, we compared patients with a history of restrictive bariatric procedures (LSG and LAGB) with patients having a history of RYGB and patients without a history of obesity surgery.

\section{Statistical analysis}

Results are presented as median values (interquartile range) for continuous variables, and numbers (percentages) for categorical variables. The $\chi^{2}$ test or Fisher's exact test was used for categorical variables, and unpaired $t$-test for continuous variables. A two-sided significance level of 0.05 was used for all statistical tests. Analyses were performed using SAS v. 9.4 (SAS Institute, Cary, NC, USA).

\section{Results}

Of the 13,242 patients who underwent TT from 2006 to $2018,0.7 \%(n=90)$ had a history of bariatric surgery and were included in the study; the number of patients with a history of bariatric surgery who underwent TT (mainly LSG and RYGB) steadily increased in the last years of the study period (Figure 1). Among these, $38.8 \%$ of patients $(\mathrm{n}=35)$ underwent LAGB, 32.2\% underwent LSG $(\mathrm{n}=29)$, and $30 \%$ underwent RYGB ( $\mathrm{n}=26)$. The median interval between bariatric surgery and thyroidectomy was 4 years (range, 2-15 years). The median BMI at the time of TT was $30.9 \mathrm{~kg} / \mathrm{m}^{2}$ (range, $20.2-51.4 \mathrm{~kg} / \mathrm{m}^{2}$ ). Overall, $92 \%(\mathrm{n}=83)$ of the patients were women and the median age was 49.2 years (range, 23.1-69.4 years). The three groups presented with no differences in the preoperative clinical and biochemical characteristics (specifically the serum calcium and vitamin D levels) (Table 1). There were no differences in the percentage of thyroid surgery for malignancy.

No patient was lost to follow-up. The median followup period was 4 years (range, 1-7 years). Twenty-five patients $(27.8 \%)$ presented with immediate postoperative hypocalcemia. Patients with a history of RYGB were more likely to develop postoperative hypocalcemia $(50 \% ; n=13)$ than patients with a history of LAGB $(17.1 \%$; $=6)$ or LSG $(20.6 \%, \mathrm{n}=6)(\mathrm{P}=0.003$; Figure 2).

Calcium, in the form of calcium gluconate, was supplemented intravenously to six patients; one belonged to the LAGB patients, while five belonged to the RYGB patients. Permanent hypoparathyroidism was observed in one patient with a history of LAGB and 5 with a history of RYGB, however, it was not observed in the LSG group. We found no significant differences in the rates of hypocalcemia, both transient and permanent, between patients with a history of restrictive procedures and patients without a history of bariatric surgery $[18.8 \%(12 / 64) v s$. $17.2 \%(2,268 / 13,152), \mathrm{P}=0.4]$ (Table 2). 
Table 1 Patients demographic characteristics

\begin{tabular}{|c|c|c|c|c|c|}
\hline Characteristics & No previous bariatric surgery & LAGB & LSG & RYGB & $P$ value \\
\hline Sex, male & $2,681(20.4)$ & $4(11.4)$ & $1(3.4)$ & $2(7.7)$ & 0.3 \\
\hline Age, years & $52.1(11.8-90.0)$ & $54(25.6-63.9)$ & $48.1(23.1-69.4)$ & $49(34.9-64.8)$ & 0.8 \\
\hline $\mathrm{BMI}$ at time to thyroid surgery, $\mathrm{kg} / \mathrm{m}^{2}$ & $24.8(13.3-61.0)$ & $32.7(20.2-51.4)$ & $31.6(23.1-44.2)$ & $29.3(20.4-48.8)$ & 0.01 \\
\hline \multicolumn{6}{|l|}{ Preoperative biochemical results } \\
\hline Calcium level, mg/dL & $9.3(8.9-11.2)$ & $9.3(9.0-10.5)$ & $9.4(9.0-10.2)$ & $9.6(8.9-11.4)$ & 0.3 \\
\hline 25-hydroxy-vitamin D, ng/mL & $31[21-47]$ & $35[22-47]$ & $31[25-40]$ & 29 [21-44] & 0.1 \\
\hline
\end{tabular}

Data are presented as $\mathrm{n}$ (\%) or median (interquartile range). LAGB, laparoscopic gastric banding; LSG, laparoscopic sleeve gastrectomy; RYGB, Roux-en-Y gastric bypass; BMI, body mass index.

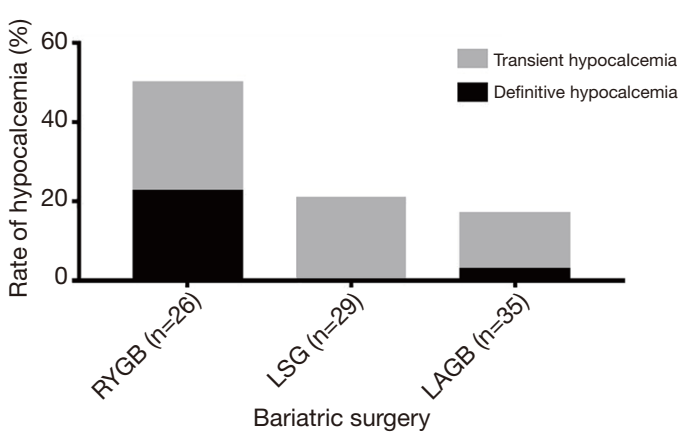

Figure 2 Hypocalcemia rate in patients with a history of bariatric surgery who underwent TT. LAGB, laparoscopic gastric banding; LSG, laparoscopic sleeve gastrectomy; RYGB, Roux-en-Y gastric bypass; TT, total thyroidectomy.

\section{Discussion}

According to the global registry report 2018 (International Federation for the Surgery of Obesity and metabolic disorders), bariatric surgery is on the rise worldwide (21). While different surgical procedures are available, currently, LSG and RYGB are the most commonly performed $(21,22)$.

Many authors believe that LSG has similar mid- and long-term outcomes in terms of weight loss and resolution of obesity-related comorbidities when compared to RYGB (23-25); however, this is debatable (26,27). LSG is based on restriction and hormonal changes that may affect hunger and satiety, and not on malabsorption, thereby causing fewer nutritional or vitamin deficiencies (23).
Our findings show that patients undergoing restrictive procedures such as LSG or LAGB carry the same risk for post-thyroidectomy hypocalcemia as patients without a history of bariatric surgery; however, patients with a history of RYGB show an increased rate of this complication.

One possible explanation could be the malabsorption due to the modified anatomy of RYGB: the duodenum and the proximal jejunum have the highest concentration of calcium transporters, and are thus, the main absorption sites of calcium (28). After gastric bypass surgery, the food no longer passes through these portions of the bowel, resulting in calcium and vitamin $\mathrm{D}$ deficiencies $(16,18)$.

Several authors have reported that gastric bypass patients who undergo TT have a higher rate of recalcitrant, symptomatic hypocalcemia and increased postoperative requirements of intravenous calcium, which leads to a prolonged hospital stay (15-19). Different strategies have been evaluated to prevent hypocalcemia in bariatric patients undergoing TT.

Manco et al. suggested that candidates for obesity surgery should be screened for thyroid dysfunction; if thyroid surgery is necessary, screening should be performed before the bariatric procedure (29). Controversy remains whether obesity increases intra- and postoperative morbidity. A recent prospective analysis did not reveal higher complication rates, despite a longer duration of the procedure (30). This finding conflicts with previous studies advocating that bariatric surgery should be performed first $(31,32)$. We believe that it is of utmost importance that 
Table 2 Hypocalcemia following TT

\begin{tabular}{|c|c|c|c|c|c|}
\hline Variables & No previous bariatric surgery & LAGB & LSG & RYGB & $P$ value \\
\hline Hypocalcemia & $2,268(17.2)$ & $6(17.1)$ & $6(20.7)$ & $13(50.0)$ & 0.003 \\
\hline Transient & - & 5 & 6 & 7 & \\
\hline Permanent & - & 1 & 0 & 6 & \\
\hline
\end{tabular}

Data are presented as $\mathrm{n}(\%)$ or median (interquartile range). *, lowest concentration of calcium after thyroidectomy. LAGB, laparoscopic gastric banding; LSG, laparoscopic sleeve gastrectomy; RYGB, Roux-en-Y gastric bypass; TT, total thyroidectomy.

TT in these patients be performed in high volume centers specialized in endocrine surgery, in order to minimize the risk of complications such as hypoparathyroidism $(11,33)$. Some useful tools during surgery could be considered, for a more meticulous parathyroid identification, such as indocyanine green angiography which assesses intraoperative parathyroid viability and guides autotransplantation (34).

In clinical practice, we may face two situations: (I) a patient candidate to bariatric surgery with a thyroid disease requiring TT and (II) a patient with a history of bariatric surgery who requires TT. Based on our results, we have considered a possible strategy for the management of these situations; however, further studies are mandatory to evaluate its clinical efficacy.

In the first situation, we suggest that TT be performed before RYGB for either malignant or benign thyroid diseases. Therefore, we could select patients unsuitable for RYGB, in case they developed permanent post-TT hypocalcemia. These patients could be rescheduled for restrictive procedures such as LSG; in our study, such patients showed similar results as the general population in terms of hypocalcemia. In obese candidates to a restrictive surgery, the bariatric procedure could be performed first in order to reduce the technical challenges of TT in these.

In the second situation, if thyroidectomy is required in a patient with a history of bariatric surgery, TT can be performed with no additional risk of hypocalcemia, provided that the patient underwent a restrictive procedure. For patients with a history of RYGB, the extent of thyroid surgery should be evaluated carefully, and lobectomy or subtotal thyroidectomy should be preferred when possible. However, this is only a clinical suggestion and is not yet supported by current scientific literature. Further studies are still need, and this suggestion struggles to present as a clinical algorithm. Evidence-based guidelines might be possible one day with the common effort and experience of bariatric and endocrine surgeons, defining metabolic surgery and with big data collecting and sharing systems.

Although patients with a history of bariatric surgery represent a minority of the cases who undergo TT $(0.7 \%$ in our series), we believe that considering the increasing prevalence of bariatric surgeries worldwide, these patients should receive a greater focus. Hypocalcemia in RYGB patients is a clinical challenge since it might be recalcitrant to oral calcium and/or vitamin D supplementation.

There are some limitations to our study due to its observational and retrospective nature. We lack certain data such as the preoperative PTH levels, nutritional parameters, node dissection, presence of parathyroid in the specimen, and parathyroid auto-transplantation, which could be important in the interpretation of our results. Our results were not adjusted for other risk factors for postoperative hypocalcemia such as thyroid cancer stage nor percentage of Graves's disease. However, to the best of our knowledge, this is the largest series of bariatric patients undergoing TT in literature. Moreover, this cohort of patients represents a homogeneous group who underwent a standardized surgical procedure for thyroid diseases from 2006 to 2018, thereby eliminating the variability in surgical technique and approach. We believe that our findings could be a useful tool for endocrine surgeons performing TT in RYGB patients, as a reminder of a more carefully evaluated surgical indication and/or a more aggressive perioperative supplementation protocol.

Future studies are required to confirm the strength of our preliminary findings and our clinical suggestions. Big data collecting and sharing, such as EUROCRINE and BAETS databases are of the utmost importance in identifying 
prognostic factors of hypocalcemia in this specific cohort.

\section{Conclusions}

In conclusion, RYGB is a risk factor for postoperative hypocalcemia after TT, although restrictive bariatric procedures are not. An accurate preoperative assessment and supplementation of calcium and vitamin D deficiency in RYGB patients undergoing $\mathrm{TT}$ is paramount in preventing postoperative hypocalcemia. Further evaluation in prospective studies and big data gathering is necessary.

\section{Acknowledgments}

Funding: None.

\section{Footnote}

Reporting Checklist: The authors have completed the STROBE reporting checklist. Available at https://dx.doi. org/10.21037/gs-21-225

Data Sharing Statement: Available at https://dx.doi. org/10.21037/gs-21-225

Peer Review File: Available at https://dx.doi.org/10.21037/ gs-21-225

Conflicts of Interest: All authors have completed the ICMJE uniform disclosure form (available at https://dx.doi. org/10.21037/gs-21-225). The authors have no conflicts of interest to declare.

Ethical Statement: The authors are accountable for all aspects of the work in ensuring that questions related to the accuracy or integrity of any part of the work are appropriately investigated and resolved. The study was conducted in accordance with the Declaration of Helsinki (as revised in 2013). The study was approved by institutional ethics board of Assistance Publique-Hôpitaux de Paris (APHP) (IRB number 20200115171338) and informed consent was taken from all the patients.

Open Access Statement: This is an Open Access article distributed in accordance with the Creative Commons Attribution-NonCommercial-NoDerivs 4.0 International License (CC BY-NC-ND 4.0), which permits the noncommercial replication and distribution of the article with the strict proviso that no changes or edits are made and the original work is properly cited (including links to both the formal publication through the relevant DOI and the license). See: https://creativecommons.org/licenses/by-nc-nd/4.0/.

\section{References}

1. Flegal KM, Kruszon-Moran D, Carroll MD, et al. Trends in Obesity Among Adults in the United States, 2005 to 2014. JAMA 2016;315:2284-91.

2. WHO. Obesity and overweight; 2018. Available online: http://www.who.int/mediacentre/factsheets/fs311/en/

3. Colquitt JL, Pickett K, Loveman E, et al. Surgery for weight loss in adults. Cochrane Database Syst Rev 2014;(8):CD003641.

4. Antoniewicz A, Kalinowski P, Kotulecka KJ, et al. Nutritional Deficiencies in Patients after Roux-en-Y Gastric Bypass and Sleeve Gastrectomy during 12-Month Follow-Up. Obes Surg 2019;29:3277-84.

5. Cole AJ, Beckman LM, Earthman CP. Vitamin D status following bariatric surgery: implications and recommendations. Nutr Clin Pract 2014;29:751-8.

6. Bacci V, Silecchia G. Vitamin D status and supplementation in morbid obesity before and after bariatric surgery. Expert Rev Gastroenterol Hepatol 2010;4:781-94.

7. Slater GH, Ren CJ, Siegel N, et al. Serum fat-soluble vitamin deficiency and abnormal calcium metabolism after malabsorptive bariatric surgery. J Gastrointest Surg 2004;8:48-55; discussion 54-5.

8. Chakhtoura MT, Nakhoul NN, Shawwa K, et al. Hypovitaminosis D in bariatric surgery: A systematic review of observational studies. Metabolism 2016;65:574-85.

9. Données CNAMTS. Available online: https://www.ameli.fr/

10. Seo GH, Chai YJ, Choi HJ, et al. Incidence of permanent hypocalcaemia after total thyroidectomy with or without central neck dissection for thyroid carcinoma: a nationwide claim study. Clin Endocrinol (Oxf) 2016;85:483-7.

11. Edafe O, Antakia R, Laskar N, et al. Systematic review and meta-analysis of predictors of post-thyroidectomy hypocalcaemia. Br J Surg 2014;101:307-20.

12. Stack BC Jr, Bimston DN, Bodenner DL, et al. American Association of Clinical Endocrinologists and American College of Endocrinology Disease State Clinical Review: Postoperative Hypoparathyroidism--Definitions and Management. Endocr Pract 2015;21:674-85.

13. Panazzolo DG, Braga TG, Bergamim A, et al. Hypoparathyroidism after Roux-en-Y gastric bypass--a 
challenge for clinical management: a case report. J Med Case Rep 2014;8:357.

14. Pietras SM, Holick MF. Refractory hypocalcemia following near-total thyroidectomy in a patient with a prior Rouxen-Y gastric bypass. Obes Surg 2009;19:524-6.

15. Durr ML, Saunders JR, Califano JA, et al. Severe hypocalcemia complicating thyroid surgery after Rouxen-Y gastric bypass procedure. Arch Otolaryngol Head Neck Surg 2009;135:507-10.

16. Goldenberg D, Ferris RL, Shindo ML, et al. Thyroidectomy in patients who have undergone gastric bypass surgery. Head Neck 2018;40:1237-44.

17. McKenzie TJ, Chen Y, Hodin RA, et al. Recalcitrant hypocalcemia after thyroidectomy in patients with previous Roux-en-Y gastric bypass. Surgery 2013;154:1300-6; discussion 1306.

18. Salinger EM, Moore JT. Profound hypocalcemia after near-total thyroidectomy in a Roux-en-Y gastric bypass patient. Am Surg 2010;76:E7-8.

19. Chereau N, Vuillermet C, Tilly C, et al. Hypocalcemia after thyroidectomy in patients with a history of bariatric surgery. Surg Obes Relat Dis 2017;13:484-90.

20. Spartalis E, Thanassa A, Athanasiadis DI, et al. Postthyroidectomy Hypocalcemia in Patients With History of Bariatric Operations: Current Evidence and Management Options. In Vivo 2019;33:1373-9.

21. Angrisani L, Santonicola A, Iovino P, et al. IFSO Worldwide Survey 2016: Primary, Endoluminal, and Revisional Procedures. Obes Surg 2018;28:3783-94.

22. Angrisani L, Santonicola A, Iovino P, et al. Bariatric Surgery and Endoluminal Procedures: IFSO Worldwide Survey 2014. Obes Surg 2017;27:2279-89.

23. Castagneto Gissey L, Casella Mariolo JR, Genco A, et al. 10-year follow-up after laparoscopic sleeve gastrectomy: Outcomes in a monocentric series. Surg Obes Relat Dis 2018;14:1480-7.

24. Felsenreich DM, Langer FB, Prager G. Weight Loss and Resolution of Comorbidities After Sleeve Gastrectomy: A Review of Long-Term Results. Scand J Surg 2019;108:3-9.
25. Shoar S, Saber AA. Long-term and midterm outcomes of laparoscopic sleeve gastrectomy versus Roux-en-Y gastric bypass: a systematic review and meta-analysis of comparative studies. Surg Obes Relat Dis 2017;13:170-80.

26. Ahmed B, King WC, Gourash W, et al. Long-term weight change and health outcomes for sleeve gastrectomy (SG) and matched Roux-en-Y gastric bypass (RYGB) participants in the Longitudinal Assessment of Bariatric Surgery (LABS) study. Surgery 2018;164:774-83.

27. Golzarand M, Toolabi K, Farid R. The bariatric surgery and weight losing: a meta-analysis in the long- and very long-term effects of laparoscopic adjustable gastric banding, laparoscopic Roux-en-Y gastric bypass and laparoscopic sleeve gastrectomy on weight loss in adults. Surg Endosc 2017;31:4331-45.

28. Johnson JM, Maher JW, DeMaria EJ, et al. The long-term effects of gastric bypass on vitamin D metabolism. Ann Surg 2006;243:701-4; discussion 704-5.

29. Manco M, Nanni G, Tondolo V, et al. Hypocalcemia complicating near-total thyroidectomy in patients with coexisting lipid malabsorption due to biliopancreatic diversion. Obes Surg 2004;14:1429-34.

30. Blanchard C, Bannani S, Pattou F, et al. Impact of body mass index on post-thyroidectomy morbidity. Head Neck 2019;41:2952-9.

31. Milone M, Musella M, Conzo G, et al. Thyroidectomy in high body mass index patients: A single center experience. Int J Surg 2016;28 Suppl 1:S38-41.

32. Finel JB, Mucci S, Branger F, et al. Thyroidectomy in patients with a high BMI: a safe surgery? Eur J Endocrinol 2014;171:99-105.

33. Orloff LA, Wiseman SM, Bernet VJ, et al. American Thyroid Association Statement on Postoperative Hypoparathyroidism: Diagnosis, Prevention, and Management in Adults. Thyroid 2018;28:830-41.

34. Rudin AV, McKenzie TJ, Thompson GB, et al. Evaluation of Parathyroid Glands with Indocyanine Green Fluorescence Angiography After Thyroidectomy. World J Surg 2019;43:1538-43.
Cite this article as: Cali B, Hasani A, Buffet C, Menegaux F, Chereau N. Is there a relationship between different types of prior bariatric surgery and post-thyroidectomy hypocalcemia? Gland Surg 2021;10(7):2088-2094. doi: 10.21037/gs-21-225 